Introduction: Acute kidney injury (AKI) is a frequent postoperative complication. However, data on the incidence of AKI in patients with colorectal cancer (CRC) undergoing surgery with curative intent are still limited. We examined the relationship between postoperative AKI among CRC surgery patients and preoperative therapy or no prior therapy.

Material and methods: A total of 326 consecutive patients from the regional oncology center undergoing CRC surgery in the period January to December 2019 were included in the observational cohort study. We defined $\mathrm{AKI}$ as a $50 \%$ increase in plasma creatinine or initiation of renal replacement therapy within 7 days after surgery or an absolute increase in creatinine of $0.3 \mathrm{mg} / \mathrm{dl}$ within 48 hours.

Results: Acute kidney injury occurred in 36 patients (11\%), 27 of whom underwent rectum resection, and 9 underwent colon resection. The incidence of AKI was identical in both types of surgery. Among 54 patients undergoing neoadjuvant radiochemotherapy, 6 patients $(11 \%)$ developed AKI, while there was no case of AKI in 31 patients with neoadjuvant radiotherapy. Among 36 patients with AKI, 33 had hypertension, 27 had diabetes and 18 had at least stage 3 of chronic kidney disease before the surgery.

Conclusions: Acute kidney injury after surgery for CRC is a relatively frequent postoperative complication, in particular, in patients with prior impairment in kidney function and comorbidities such as hypertension and diabetes. Appropriate preoperative therapy, including optimal hydration, withdrawal of potentially nephrotoxic drugs, etc., may reduce the incidence of AKI.

Key words: acute kidney injury, colorectal cancer, chronic kidney disease, surgery.

Contemp Oncol (Pozn) 2021; 25 (4): 187-190 DOI: https://doi.org/10.5114/wo.2021.111057

\section{Acute kidney injury prevalence in patients with colorectal cancer undergoing surgery with curative intent}

\author{
Leszek Kozlowski ${ }^{1}$, Jolanta Malyszko²
}

${ }^{1}$ Department of Oncological Surgery, Bialystok Cancer Center, Poland ${ }^{2}$ Department of Nephrology, Dialysis and Internal Medicine, Medical University of Warsaw, Poland

\section{Introduction}

Patients presenting with malignancies have a high risk of developing acute kidney injury (AKI) secondary to receiving chemotherapy, exposure to contrast agents used in medical imaging, radiation therapy, tumor lysis syndrome, hypotension or caused by the direct effects of the malignancy. Acute kidney injury is a frequent complication in cancer patients and is associated with increased morbidity and mortality. A Danish population based cohort study reported the incidence of $\mathrm{AKI}$ in cancer patients followed up for more than 7 years [1]. Of 1.2 million individuals, 44,116 developed a malignancy. The risk of AKI was $17.5 \%$ during the first year after cancer diagnosis and the overall 5-year risk of AKI was $27.0 \%$. The highest incidence rates were in patients with cancer of the kidney (44\%), biliary tract, liver, and pancreas, and in patients with multiple myeloma. Surgery with curative intent plays a crucial role in the treatment of colorectal cancer (CRC). A small cohort study included 288 medical records from elective rectal cancer surgery and found an AKI occurrence rate of 3.8\% [2]. Studies of other major abdominal procedures reported postoperative AKI in 3-35\% of patients [3], and found AKI to be associated with increased mortality [4]. In another study from a single tertiary hospital in Korea, AKI occurred in 177 (25.9\%) during the first postoperative year, mainly during the first 3 months [5].

Taking all these facts into consideration and limited data on the AKI prevalence in the early postoperative period, we aimed to assess the relationship between postoperative AKI among CRC surgery patients and in relation to neoadjuvant therapy or no prior therapy [5].

\section{Material and methods}

A total of 326 consecutive patients from the regional oncology center undergoing CRC surgery with curative intent in the period from January to December 2019 were included in the retrospective, observational cohort study. We defined AKI as a 50\% increase in plasma creatinine or initiation of renal replacement therapy within 7 days after surgery or an absolute increase in creatinine of $0.3 \mathrm{mg} / \mathrm{dl}$ within 48 hours using KDIGO (Kidney Disease: Improving Global Outcomes) guidelines [6]. Electronic medical records of the included patients were retrospectively reviewed. Demographic and clinical data at the time of admission for surgery including age, sex, body mass index (BMI), underlying disease (hypertension, diabetes, and cardiovascular disease), and types of surgery (rectal resection, colon resection) were recorded as well as laboratory data including hemoglobin, albumin, total cholester$\mathrm{ol}$, urea, and creatinine levels, and the estimated glomerular filtration rate (eGFR). Blood pressure was measured with standard protocols. Hypertension was defined as either a blood pressure of at least 140/90 mm Hg or hypo- 
Table 1. Clinical and biochemical data of colorectal cancer patients with and without acute kidney injury

\begin{tabular}{|c|c|c|c|}
\hline Parameters & $\begin{array}{l}\text { No AKI } \\
n=54\end{array}$ & $\begin{array}{c}\text { AKI } \\
n=46\end{array}$ & $p$-value \\
\hline Age [years] & $66.87 \pm 9.87$ & $72.89 \pm 8.48$ & 0.0005 \\
\hline Albumin $[\mathrm{g} / \mathrm{l}]$ & $42.82 \pm 3.70$ & $42.92 \pm 3.71$ & 0.9707 \\
\hline Sodium $[\mathrm{mmol} / \mathrm{l}]$ & $139.77 \pm 2.57$ & $139.35 \pm 2.72$ & 0.5677 \\
\hline Potassium [mmol/l] & $4.47 \pm 0.38$ & $4.96 \pm 0.41$ & 0.0000 \\
\hline Hematocrit [\%] & $35.73 \pm 5.10$ & $33.06 \pm 5.57$ & 0.0639 \\
\hline Hemoglobin $[\mathrm{g} / \mathrm{l}]$ & $122.0 \pm 18.1$ & $110.1 \pm 21.1$ & 0.0237 \\
\hline Erythrocyte count [x 1012/l] & $4.28 \pm 0.37$ & $3.89 \pm 0.57^{\star}$ & 0.0048 \\
\hline $\operatorname{MCV}[\mathrm{fl}]$ & $82.99 \pm 10.94$ & $84.91 \pm 9.64$ & 0.5212 \\
\hline $\mathrm{MCH}[\mathrm{pg}]$ & $28.56 \pm 3.64$ & $28.29 \pm 4.08$ & 0.7729 \\
\hline RDW [\%] & $7.23 \pm 1.30$ & $7.23 \pm 0.76$ & 0.9932 \\
\hline Leukocyte count $\left[10^{9} / l\right]$ & $6.78 \pm 2.32$ & $6.44 \pm 1.49$ & 0.5481 \\
\hline Platelet count $\left[10^{9} / l\right]$ & $271.71 \pm 82.58$ & $239.50 \pm 44.58$ & 0.1563 \\
\hline Creatinine $[\mu \mathrm{mol} / \mathrm{l}]$ & $86.63 \pm 15.80$ & $102.54 \pm 32.71^{\star * *}$ & 0.0000 \\
\hline Urea $[\mathrm{mmol} / \mathrm{l}]$ & $12.61 \pm 5.64$ & $18.10 \pm 4.69^{\star \star \star}$ & 0.0000 \\
\hline eGFR by CKD-EPI [ml/min/1.72 m²] & $87.95 \pm 18.74$ & $66.32 \pm 21.98^{\star \star \star}$ & 0.0000 \\
\hline $\mathrm{PT}[\mathrm{s}]$ & $12.43 \pm 0.99$ & $12.01 \pm 0.71$ & 0.1265 \\
\hline INR & $1.05 \pm 0.09$ & $1.01 \pm 0.06$ & 0.1069 \\
\hline Total protein [g/l] & $67.71 \pm 5.50$ & $69.01 \pm 3.52$ & 0.3871 \\
\hline Glucose $[\mathrm{mmol} / \mathrm{l}]$ & $6.10 \pm 1.49$ & $6.38 \pm 2.89$ & 0.5821 \\
\hline
\end{tabular}

AKI - acute kidney injury, CKD-EPI - chronic kidney disease epidemiology collaboration, eGFR - estimated glomerular filtration rate, INR - international normalized ratio, $M C H$ - mean cell hemoglobin, MCV - mean corpuscular volume, PT - prothrombin time, RDW - red cell distribution width

Conversion factors to SI units are as follows: for glucose - 0.0555, for creatinine 88.4, for hemoglobin 10, for urea 0.357, albumin, total protein $10 .{ }^{*} p<0.05,{ }^{\star * *} p<0.001$

tensive medication use. Chronic kidney disease (CKD) was defined according to 2012 KDIGO guidelines [7] with eGFR estimated using the chronic kidney disease epidemiology collaboration (CKD-EPI) formula [8]. Preoperative therapy was also investigated. Neoadjuvant radiochemotherapy was administered to 54 patients, whereas 31 patients underwent neoadjuvant radiotherapy. No neoadjuvant therapy was administered to 241 patients prior the surgery. Patients underwent laparoscopic surgery ( 87 in rectal group and 7 in colon group) and open surgery (169 in rectal group and 63 in the colon group). 256 patients underwent rectum resection, and 70 underwent colon resection. The main methods of colon or rectal surgery performed in the department of surgery were: right hemicolectomy (58), left hemicolectomy (12), low anterior resection (185), high anterior resection (51), abdomino-perineal resection (20). Adenocarcinoma was diagnosed in all treated patients by endoscopy before qualifying for surgery. Patients' clinical staging was determined on the basis of the criteria of the International Union Against Cancer, 8th edition. The study was approved by the appropriate ethics review board and is compliant with the Declaration of Helsinki. As only retrospective medical data were analyzed, informed consent was waived.

Data were analyzed using Statistica 13.1 computer software (Tulsa, OK, USA). Normality of variable distribution was tested using the Shapiro-Wilk test. Student's t-test was used in statistical analysis to compare differences between groups with $p<0.05$ considered statistically significant, when appropriate.

\section{Results}

The clinical characteristics of the studied patients are shown in Table 1. Patients with AKI were older, with higher creatinine, urea, and potassium, and lower hemoglobin, erythrocyte count, hematocrit and eGFR. Acute kidney injury occurred in 36 patients (11\%), 27 of whom underwent rectum resection, and 9 underwent colon resection. The incidence of AKI was identical in both types of surgery. Among 54 patients undergoing neoadjuvant radiochemotherapy, 6 patients (11\%) developed AKI, while there was no case of AKI in 31 patients with neoadjuvant radiotherapy. Among 36 patients with AKI, 33 had hypertension, 27 had diabetes and 18 had at least stage 3 of CKD before the surgery.

\section{Discussion}

In our study AKI occurred in 36 (11\%) patients postoperatively; none of the patients required renal replacement therapy. Type of the surgery (rectal vs colonic resection) was not associated with AKI. Our results also are consistent with reported prevalence of 3-35\% for postoperative AKI [3]. The incidences of AKI in 6495 inpatients with GI cancers in a tertiary hospital in eastern China were $20.5 \%$, $13.9 \%$, and $12.5 \%$, respectively for esophagus, stomach, and intestine cancer as reported by Jin et al. [9]. They reported that about $50.0 \%$ of AKI cases and $18.8 \%$ of AKI deaths occurred in patients with GI cancer [9]. In other studies from China occurrence of AKI in GI cancer was reported between 2.4 and 35.3\% [10-12]. 
It may be partly explained by the heterogeneity of the source population, the different definitions for AKI as well as oncologists' neglect of AKI diagnosis. Data on perioperative AKI in cancer patients are scarce and perioperative AKI has primarily been reported in patients undergoing cardiac surgery or transplantation $[13,14]$ AKI occurs in $5-10 \%$ of all hospitalized patients, $4-13.4 \%$ of patients who have undergone major abdominal surgery, and up to $60 \%$ of intensive care unit patients $[15,16] \mathrm{Sim}$ et al. [17] reported recently that postoperative $A K I$ ranged between $8.8 \%$ in patients with CRC undergoing laparoscopic surgery and $9.1 \%$ in patients undergoing open surgery $(p=0.406)$. As reported by Kee et al. [5], AKI occurred in 25.9\% during the first postoperative year, most commonly during the first 3 months after surgery (up to 50\%). Patients who developed AKI were significantly older, with lower BMI, and significantly lower preoperative hemoglobin and serum albumin levels. Slagelse et al. [18] included 6580 patients from the Danish Colorectal Cancer Group database who underwent CRC surgery in Northern Denmark during 2005-2011 and found that AKI occurred in 1337 patients (20.3\%). In another study from Denmark, Iversen et al. [19] examined postoperative complications and mortality in 2157 patients with CRC who underwent emergency surgery. They included only patients with AKI receiving renal replacement therapy (RRT). They found that $2.0 \%$ of postoperative patients with CRC had AKI and were receiving RRT; 30-day mortality in this group was $68 \%$. On the other hand, Lim et al. [2] assessed AKI occurrence in a small cohort study of 288 patients undergoing elective rectal cancer surgery. They reported that 3.8\% of patients developed AKI. They also found that the in-hospital mortality of patients with AKI was $18.2 \%$, whereas patients without AKI had an in-hospital mortality of $0.7 \%$. In contrast to the study of Huang et al.[20], we did not find a difference in the rate of AKI between laparotomy and laparoscopic procedure groups. They studied 285 patients and postoperative AKI occurred only in 16 patients (7.5\%) from the laparotomy group $(n=212)$.

Similar to patients undergoing major abdominal surgery, patients who undergo colorectal surgery with intestinal resection are also prone to AKI, but the mechanisms of $A K I$ are different. As AKI is multifactorial, both operative setting such as response to anaesthesia with peripheral vasodilatation and myocardial depression and surgery with rise in aldosterone and antidiuretic hormone together with the effect of fluid depletion should be considered in the origin of AKI. This could be due to global hypoperfusion of the kidney if the afferent arteriole dilation and efferent arteriole vasoconstriction response initiated by the kidney does not result in adequate glomerular filtration. Further, hypotension can lead to dysfunctional intrarenal microcirculation due to patchy areas of hypoperfusion in the kidney and potentially add to the risk of developing AKI [21-23]. One of the major functions of the colon is to reabsorb water; therefore, resection of the colon may aggravate dehydration depending on the extent of resected colon. In addition to routine postoperative complications such as bleeding, infection, and anastomotic site leakage, the CRC group shows chronic salt and water loss and malabsorption. Moreover, exposure to nephrotoxic agents (chemotherapeutic and radiocontrast agents) and ostomy maintenance may also contribute to risk of AKI in this population [10, 24, 25]. In our study patients with prior radiotherapy did not develop AKI, while among subjects with prior radiochemotherapy $11 \%$ developed $\mathrm{AKI}$. In a recent review, Borucki et al. [26] reported that significant dehydration was common following ileostomy and was linked with $\mathrm{AKI}$ and had a long-term impact on renal function.

\section{Limitations of the study}

This study has several limitations. First, the retrospective observational design is a drawback. Secondly, only patients undergoing surgery with curative intent were analyzed. Thirdly, it was a single-center study from one regional oncology center. Data on potential risk factors for AKI including medication could only be identified from medical records. However, being a single center with similar surgery techniques and manner and source of data collection could be a benefit.

\section{Conclusions}

Acute kidney injury occurred in approximately $12 \%$ of patients undergoing CRC surgery. Prior radiochemotherapy and comorbidities such as hypertension and diabetes were risk factors for AKI.

Proper preoperative therapy may reduce the incidence of AKI.

\section{The authors declare no conflict of interest.}

\section{References}

1. Christiansen CF, Johansen MB, Langeberg WJ, Fryzek JP, Sørensen HT. Incidence of acute kidney injury in cancer patients: a Danish population based cohort study. Eur J Intern Med 2011; 22: 399-406.

2. Lim SY, Lee JY, Yang JH, et al. Predictive factors of acute kidney injury in patients undergoing rectal surgery. Kidney Res Clin Pract 2016; 35: 160.

3. Gameiro J, Fonseca JA, Neves M, et al. Acute kidney injury in major abdominal surgery: incidence, risk factors, pathogenesis and outcomes. Ann Intensive Care 2018; 8: 22.

4. Spanjersberg WR, Reurings J, Keus F, et al. Fast track surgery versus conventional recovery strategies for colorectal surgery. Cochrane Database Syst Rev 2011; 16: CD007635.

5. Kee YK, Kim H, Jhee JH, et al. Incidence of and risk factors for delayed acute kidney injury in patients undergoing colorectal surgery. Am J Surg. 2019; 218: 907-912.

6. Kidney Disease: Improving Global Outcomes Workgroup. KDIGO clinical practice guideline for acute kidney injury. Kidney Int 2012; 2: 1-138.

7. KDIGO 2012 Clinical Practice Guideline for the Evaluation and Management of Chronic Kidney Disease. Kidney Inter Suppl 2013; 3: 1-150.

8. Levey AS, Stevens LA, Schmid CH, et al. A new equation to estimate glomerular filtration rate. Ann Intern Med 2009; 150: 604-612.

9. Jin J, Wang Y, Shen Q, et al. Acute kidney injury in cancer patients: a nationwide survey in China. Sci Rep 2019; 9: 3540.

10. Li L, Lau KS, Ramanathan V, et al. Ileostomy creation in colorectal cancer surgery: risk of acute kidney injury and chronic kidney disease. J Surg Res 2017; 210: 204-212.

11. Wang W, Wang T, Feng $X$, et al. Incidence and risk factors of acute kidney injury after esophageal cancer surgery: a nested case-control study. Int J Surg 2017; 39: 11-15. 
12. Li Y, Chen X, Shen Z, et al. Prediction models for acute kidney injury in patients with gastrointestinal cancers: a real-world study based on Bayesian networks. Ren Fail 2020; 42: 869-876.

13. Jahangirifard A, Ahmadi ZH, Khalili N, et al. Early post-operative acute kidney injury after cardiac transplantation: incidence and predictive factors. Clin Transplant 2021; e14420.

14. Verwijmeren L, Bosma M, Vernooij LM, et al. Associations between preoperative biomarkers and cardiac surgery-associated acute kidney injury in elderly patients: a cohort study. Anesth Analg 2021; 133: 570-577.

15. O'Connor ME, Kirwan CJ, Pearse RM, Prowle JR. Incidence and associations of acute kidney injury after major abdominal surgery. Intensive Care Med 2016; 42: 521-530.

16. Lameire NH, Bagga A, Cruz D, et al. Acute kidney injury: an increasing global concern. Lancet 2013; 382: 170-179.

17. Sim JH, Kang SJ, Bang JY, Song JG. Comparison of the effects of laparoscopic and open surgery on postoperative acute kidney injury in patients with colorectal cancer: propensity score analysis. J Clin Med 2021; 10: 1438.

18. Slagelse C, Gammelager H, Iversen LH, et al. Acute kidney injury and 1-year mortality after colorectal cancer surgery: a population-based cohort study. BMJ Open 2019; 9: e024817.

19. Iversen LH, Bülow S, Christensen IJ, et al. Postoperative medical complications are the main cause of early death after emergency surgery for colonic cancer. Br J Surg 2008; 95: 1012-1019.

20. Huang S, Li Z, Zou N, et al. Comparison of postoperative acute kidney injury between laparoscopic and laparotomy procedures in elderly patients undergoing colorectal surgery. Surg Laparosc Endosc Percutan Tech 2020; 31: 160-164.

21. Calvert S, Shaw A. Perioperative acute kidney injury. Perioper Med 2012; 1: 1-11.

22. Sear JW. Kidney dysfunction in the postoperative period. $\mathrm{Br} J \mathrm{An}$ aesth 2005; 95: 20-32.

23. Carmichael P, Carmichael AR. Acute renal failure in the surgical setting. ANZ J Surg 2003; 73: 144-153.

24. Malyszko J, Tesarova P, Capasso G, Capasso A. The link between kidney disease and cancer: complications and treatment. Lancet 2020; 396: 277-287.

25. Malyszko J, Kozlowska K, Kozlowski L, Malyszko J. Nephrotoxicity of anticancer treatment. Nephrol Dial Transplant 2017; 32: 924-936.

26. Borucki JP, Schlaeger S, Crane J, Hernon JM, Stearns AT. Risk and consequences of dehydration following colorectal cancer resection with diverting ileostomy. A systematic review and meta-analysis. Colorectal Dis 2021; 23: 1721-1732.

\section{Address for correspondence}

\section{Jolanta. Małyszko}

Department of Nephrology, Dialysis and Internal Diseases

Medical University of Warsaw

la Banacha St.

02-097 Warsaw, Poland

Phone: +48225992658

Fax: +48 225991658

e-mail: jolmal@poczta.onet.pl

Submitted: 22.09.2021

Accepted: 31.10 .202 